NASA Technical Memorandum 107034

AIAA-95-2927

\title{
Hall Thruster Ion Beam Characterization
}

David H. Manzella

NYMA, Inc.

Brook Park, Ohio

and

John M. Sankovic

Lewis Research Center

Cleveland, Ohio

Prepared for the

31st Joint Propulsion Conference and Exhibit

cosponsored by AIAA, ASME, SAE, and ASEE

San Diego, California, July 10-12, 1995

National Aeronautics and

Space Administration 


\title{
Hall Thruster Ion Beam Characterization
}

\author{
David H. Manzella \\ NYMA, Inc. \\ Engineering Services Division \\ Brook Park, Ohio 44142 \\ John M. Sankovic \\ National Aeronautics and Space Administration \\ Lewis Research Center \\ Cleveland, Ohio 44135
}

The distribution of ion current density as a function of angle from the thruster axis was measured at nominal operating conditions of 300 Volts discharge voltage and 4.5 Amperes discharge current for two SPT-100 stationary plasma thrusters from Fakel Enterprises, a D-55 anode layer thruster from the Central Scientific Research Institute for Machine Building (TsNIIMASH), and a first, second, and third generation T-100 stationary plasma thruster from the Scientific Research Institute of Thermal Process (NIITP). The data showed that the current density distributions of these thrusters were similar. Some differences in peak ion current density were observed. Multiply charged ions were found to be a small fraction of the plasma plume for all of the thrusters. The effect of facility pressure on ion current density distribution was found to be nonnegligible at pressures above $2 \times 10^{-6}$ Torr. The ion current density distributions of a new SPT-100 and a 6000 hour wear tested SPT-100 exhibited no discernible difference. Ion current density measurements were also taken at off-nominal thruster operating conditions.

\section{Introduction}

International interest in Hall propulsion systems suitable for satellite propulsion has resulted in consideration of several different Russian Hall thrusters, including two different types of stationary plasma thrusters (SPT's) and the thruster with anode layer (TAL). The suitability of each of these engines for use on a spacecraft depends on performance, life, and integration issues. An investigation was undertaken to assess the density and distribution of the ion beams created by these different engines and the sensitivity of the respective beam characteristics to thruster operating conditions and test facility pressure.

Hall thrusters have a specific impulse near optimum for a number of missions including Earth orbit insertion and north-south stationkeeping (NSSK). ${ }^{1}$ In a NSSK mission the thruster ion beam may impinge on sensitive spacecraft surfaces including the anti-reflective, solar cell cover glass coatings and the conductive solar cell inter-connect material. This is depicted in Figure 1 for a geosynchronous communication satellite. The ions in the thruster plume are highly energetic. If ion impingement unacceptably degrades the performance of these components, canting of the thrusters away from the sensitive surfaces may be necessary. For an orbit raising application, a spacecraft could be configured to minimize the possibility of erosion or contamination of sensitive spacecraft surfaces due to ion impingement. For both orbit raising and NSSK applications, however, it is possible that communication signals sent to and from the spacecraft may pass through the thruster plasma plume. This may introduce phase noise to these signals or produce other communication impacts. Plume characteristics must be understood so that these issues can be avoided.

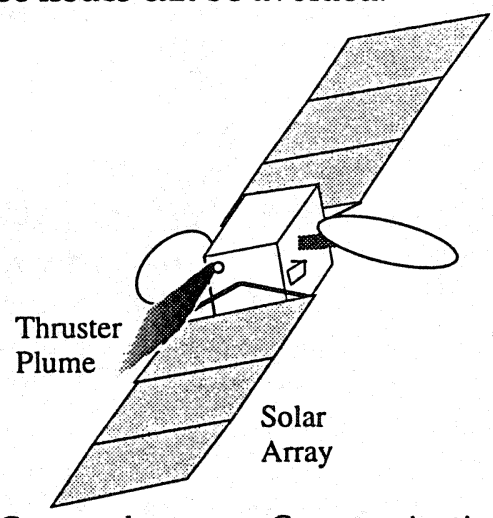

Figure 1: Geosynchronous Communication Satellite Configuration

In the past, direct measurements of the effect of ion impingement on sensitive spacecraft surfaces and communication impacts were addressed in ground based testing. 2,3 In each case, a particular geometry and thruster operating condition was considered. In order to extrapolate these results to other operating conditions and individual spacecraft geometries, an accurate description of

Copyright $(\odot 1995$ by the American Institute of Aeronautics and Astronautics, Inc. No copyright is asserted in the United States under Title 17, U.S. Code. The U.S. Government has a royalty-free license to exercise all rights under the copyright claimed herein for government purposes. All other rights are reserved by the copyright owner. 
the exhausting plasma produced by these devices is needed. For ease of implementation, this description could take the form of an empirical model. Such a model can be constructed based on experimental data taken at several thruster exit diameters from the exit plane, since the flow can be considered frozen at greater distances from the thruster. At large distances from the thruster, collisional processes are not significant, resulting in ion trajectories which are purely rectilinear, and the plume can be described as a spherical expansion from a point source.

The objective of this investigation was to measure the distribution of ion current density as a function of angle from the thrust vector for each of five different Hall thrusters at both nominal operating conditions and off nominal operating conditions. These data will provide spacecraft integrators the information required to extrapolate previous measurements to other configurations. Additionally, these data may provide insight into the operational similarities and differences between the various thrusters and the underlying physical processes governing their operation.

\section{Apparatus and Procedure}

Six Russian built $1.5 \mathrm{~kW}$ class, xenon Hall thrusters of three different types were used for this investigation. A summary of the thrusters tested is shown in Table 1. The thrusters included two different SPT-100 stationary plasma thrusters from Fakel Enterprise, the D-55 anode layer thruster from the Central Scientific Research Institute for Machine Building (TsNIIMASH), and the first, second, and third generation T-100 stationary plasma thrusters from the Scientific Research Institute of Thermal Process (NIITP). The flight quality SPT-100 thruster was described in detail elsewhere. ${ }^{1}$ The first SPT-100 thruster tested was in new condition, and was tested following a performance evaluation. ${ }^{5}$ The second SPT-100 was tested following completion of a 6000 hour wear test. $^{6}$ The SPT-100 configuration had an internal flow splitter which precluded varying the cathode flow fraction during these tests.

Table 1: Thrusters Used in this Investigation

\begin{tabular}{lcc}
\hline Thruster & Manufacturer & Comments \\
\hline SPT-100 & Fakel & new \\
SPT-100 & Fakel & wear tested \\
TAL D-55 & TsNIIMASH & lab model \\
T-100-1 & NIITP & 1st generation \\
T-100-2 & NIITP & 2nd generation \\
T-100-3 & NIITP & 3rd generation \\
\hline
\end{tabular}

The TAL D-55 was a laboratory model thruster previously investigated. ${ }^{7}$ The laboratory model cathode supplied with this thruster required approximately five times the xenon flow of the other thruster cathodes. Three T-100 stationary plasma thrusters were used. They were first, second, and third generation versions of a thruster built by NIITP. This thruster appeared to be very similar to the SPT-100. Differences between the various versions of this thruster were not externally visible and were thought to be changes in the magnetics. The nominal operating conditions and performance for each type of thruster are shown in Table 2.

Table 2: Nominal Thruster Performance

\begin{tabular}{lccc}
\hline \hline Thruster & SPT-100 & D-55 & T-100 \\
\hline Thrust, mN & 84.9 & 82.0 & 82.4 \\
Discharge Voltage, Volts & 300 & 300 & 300 \\
Discharge Current, Amperes & 4.5 & 4.2 & 4.5 \\
Power, Watts & 1350 & 1300 & 1390 \\
Anode Flow Rate, mg/s & 4.99 & 4.52 & 4.93 \\
Cathode Flow Rate, mg/s & 0.38 & 2.10 & 0.41 \\
Specific Impulse, Seconds & 1610 & 1266 & 1570 \\
Specific Impulse, Seconds ${ }^{+}$ & 1735 & 1850 & 1705 \\
Efficiency, \% & 49.7 & 39.3 & 47.1 \\
Efficiency, \% & 53.5 & 57.2 & 49.5 \\
\hline
\end{tabular}

t excluding cathode flow

In the majority of tests, commercially available power supplies were used to run the thrusters. A constant voltage supply with an in-line $10 \mathrm{Ohm}$ ballast resistor was used to run the discharge. A 2.5 $\mu \mathrm{F}$ capacitor was placed across the discharge to reduce the effect of plasma oscillations on the discharge supply. Separate dc supplies were used for the electromagnets and cathode heater, if required. A high voltage, low current supply was used to initiate thruster operation. The entire electrical system was allowed to float relative to ground. Typical cathode to ground voltages were -20 Volts. The SPT- 100 was also operated using a flight type power processing unit designed and built by Space Systems Loral ${ }^{8}$ which performed all the above mentioned functions. The choice of power supply had no effect on the measured steady-state ion current density distribution. The thrusters were run on commercially available research grade xenon having a purity better than $99.9995 \%$.

The ion current density was measured using a circular molybdenum probe with an area of 3.35 square centimeters. The probe was mounted on a $0.6 \mathrm{~m}$ arm rotated by a stepper motor driven rotary actuator. The probe was biased to -15 Volts, and surrounded by a molybdenum ring independently 
biased to -15 Volts. This was done to minimize edge effects. The ion current collected by the probe was measured as the voltage across a 24 Ohm load resistor by a 10 bit analog-to-digital data acquisition board within a micro computer. This computer was also used to control the stepper motor driven rotary actuator.

Data were taken in steps of one degree from -100 degrees to 100 degrees with respect to the thruster axis in the plane of the cathode. The cathode was located on the side corresponding to positive angles as shown in Figure 2. At each location 2000 measurements were taken and averaged prior to moving to the next angular position. The arc length separating the measurement points was approximately one centimeter. Because the diameter of the ion collector was approximately two centimeters, each measurement represents an average over two degrees centered at the angular position of the measurement.

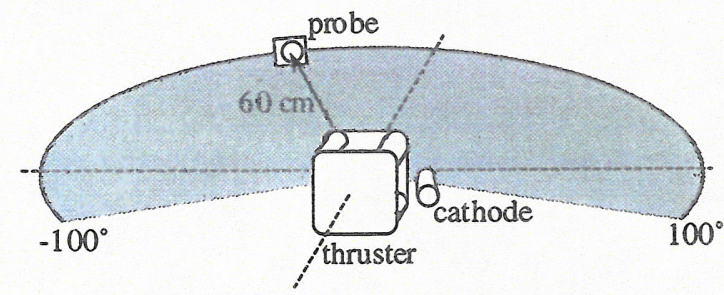

Figure 2: Apparatus for Ion Current Determination

Prior to taking ion current density measurements the thruster being tested was allowed to reach thermal equilibrium, which took on the order of tens of minutes. An entire scan from -100 to 100 degrees took approximately 15 minutes.

Data were taken in this manner in two different vacuum facilities. The majority of the data were taken in a medium sized chamber. This $5 \mathrm{~m}$ long by $1.5 \mathrm{~m}$ diameter cylindrical chamber was pumped by four $0.82 \mathrm{~m}$ oil diffusion pumps, a lobe type mechanical blower, and two piston type roughing pumps. The thruster was mounted in the center of the chamber, firing along the major axis. The ambient pressure, measured by an ionization gauge during testing, was $2 \times 10^{-5}$ Torr. In some cases, as noted, data were taken in a larger chamber. This $19 \mathrm{~m}$ long by $5 \mathrm{~m}$ diameter cylindrical chamber was cryogenically pumped with a $27 \mathrm{~m}^{2}$ helium cryopanel. The thruster was mounted in the main body of the tank, firing along the major axis of the tank. The ambient pressure measured along the chamber wall near the thruster was $2 \times 10^{-6}$ Torr unless intentionally increased by introducing additional xenon flow into the tank through an auxiliary bleed valve located on the tank wall.

\section{Results and Discussion}

Measurements of the ion current density distribution were made as a function of angle for each of the six thrusters. Measurements were taken with the thrusters operating at their nominal operating condition (4.5 Amperes discharge current and 300 Volts discharge voltage) and operating at off-nominal conditions.

\section{Nominal Operating Condition Data:}

Figure 3 shows the distribution of ion current density measured for each of the three different types of thrusters at the nominal operating point on a logarithmic and a linear scale. Linear interpolation was used between the data points taken at every degree. These data indicate that the plume profiles for each of these thrusters are similar. The only statistically significant differences are found in the peak ion current densities.

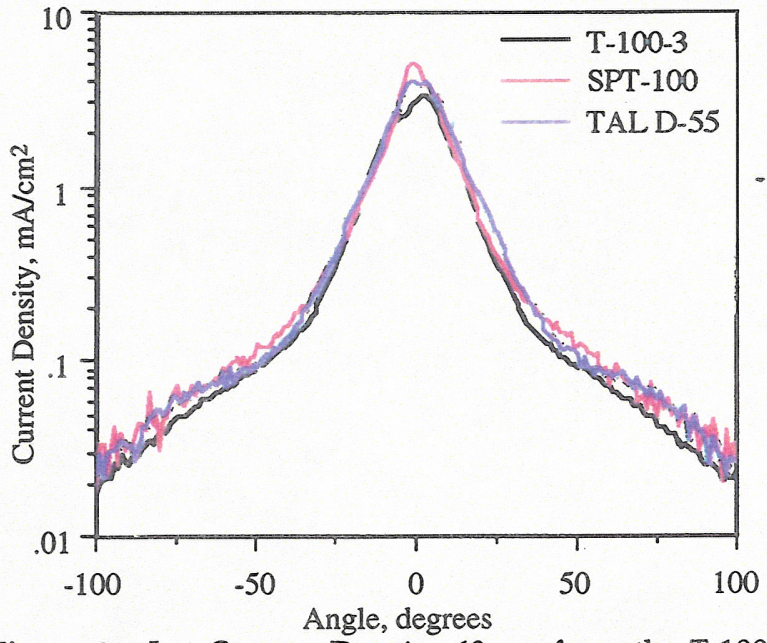

Figure 3a: Ion Current Density $60 \mathrm{~cm}$ from the T-100-3, SPT-100, and D-55 Exit Plane (Log Scale)

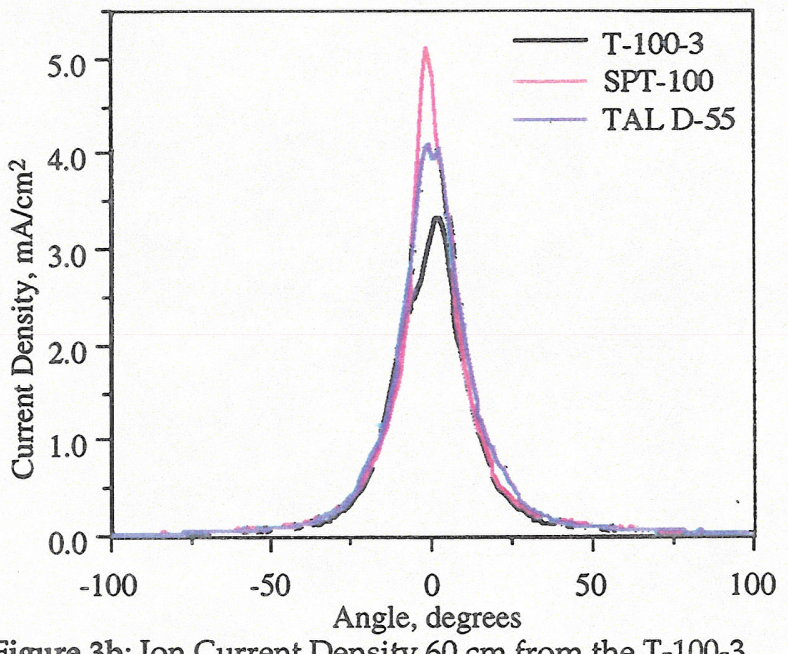

Figure 3b: Ion Current Density $60 \mathrm{~cm}$ from the T-100-3, SPT-100, and D-55 Exit Plane (Linear Scale) 
There is a central region of the plume at angles between approximately 30 and -30 degrees where the ion current density is sharply peaked. Outside of this central core the ion density drops gradually. For all thrusters, measurable ion currents were observed at angles greater than 90 degrees with respect to the thruster axis. Because there is no line of sight into the thruster at these large angles, this indicates that ions are collisionally scattered outside the thruster.

Integration of the ion current density distribution resulted in the total ion beam current. For the data shown in Figure 3 the measured ion beam current is 4.1, 4.6, and 4.9 Amperes for the T-100, SPT-100 and D-55, respectively. The differences are attributed to the differences in ion density at small angles. Details of the integration are included in an Appendix.

The total ion beam current that would be measured if each xenon atom was singly ionized can be calculated based on the measured xenon flow rate through the anode. Cathode flow was not included in this calculation for two reasons. The ionization fraction for the cathode flow in all cases was assumed to be low and the cathode flow for the TAL was disproportionately high, making comparisons with this thruster, including the cathode flow, difficult to interpret. The ion beam current calculated in this manner was 3.6, 3.7, and 3.6 for the T-100, SPT-100 and D-55, respectively. The total measured ion beam current was larger than the calculated value in all cases, even if the cathode flow was included. This was attributed to the presence of multiply charged ions. Assuming that all the multiply charged ions were doubly ionized, this indicated $13 \%, 25 \%$, and $37 \%$ doubly charged ions for the T-100, SPT-100 and D-55. Past spectroscopic studies of the SPT-100 have suggested that the fraction of doubly charged ions was on the order of $20 \%$ for the SPT- 100.9

The data taken using the SPT-100, operating at nominal conditions, were normalized to a distance of 1 meter from the thruster using a $1 / r^{2}$ dependence. Comparison of these data with similar data taken at NASA Jet Propulsion Laboratory and at Fakel Enterprises are shown in Figure 4. This was done to verify the claimed $1 / r^{2}$ current density dependence with distance from the thruster and to compare these data with data from previous investigations. The agreement between these data indicates that this normalization is valid.

It should also be noted that there was no discernible difference between the SPT data shown in Figure 4, taken with a "new" thruster, and data taken with the wear tested thruster with a total accumulated run time of 6000 hours. There .were significant differences in the size and shape of the discharge chambers near the exit of these two thrusters due to erosion of the insulating walls of the discharge chamber of the wear tested thruster. This implied that the ion current density distribution was determined upstream of this region.

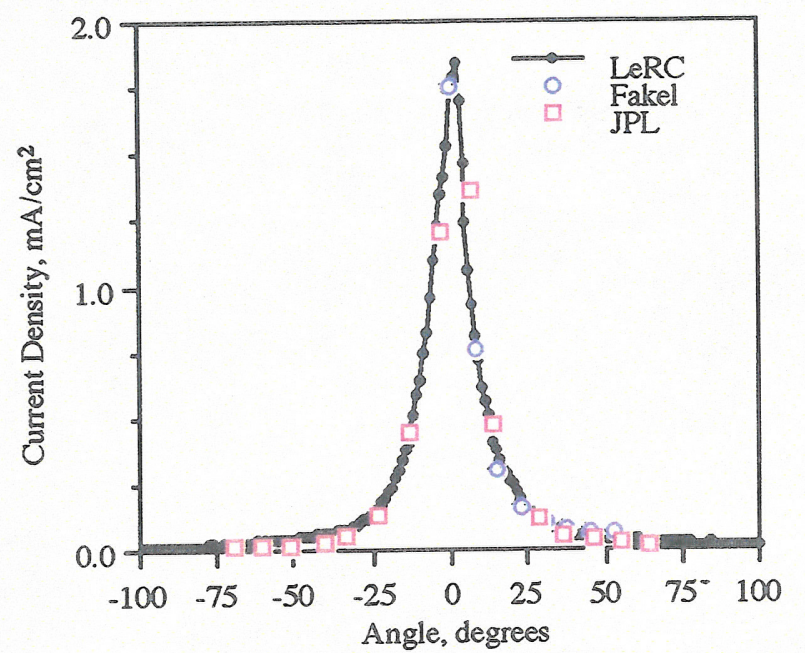

Figure 4: Ion Current Density Distribution for SPT-100 Normalized to $1 \mathrm{~m}$ from the Exit Plane

This conclusion was further supported by consideration of the potential distribution within the discharge chamber and the average ion energy as determined based on the either the measured thrust or direct measurement. ${ }^{10}$ The average ion energy determined by either of these methods was approximately $200 \mathrm{eV}$. Based on scaled floating potential measurements taken in the discharge channel of a SPT-70 with a 200 Volt discharge voltage ${ }^{11}$ it was determined that in order for an ion to be accelerated through a 200 Volt potential it would need to be created upstream of the primary erosion region of the discharge chamber.

The effect facility pressure had on the ion beam currrent density distribution was investigated by running the SPT-100 in the large testbed facility. The base operating pressure was $2 \times 10^{-6}$ Torr. For evaluation of the effect of facility pressure, the facility pressure was increased using a xenon purge. The effect of increasing pressure on the ion current density distribution is shown in Figure 5. All measurements were taken with a constant thruster flow rate. At a given facility pressure, a constant thruster flow rate ensured a constant discharge current. As the facility pressure was increased, however, the discharge current also increased. 
This effect, which resulted in a $6 \%$ increase in discharge current at $6 \times 10^{-5}$ Torr, was attributed to the ingestion of neutral xenon into the discharge chamber, where it was subsequently ionized and accelerated, providing an increase in the effective mass flow rate consisting of the supplied anode xenon flow and the ingested xenon flow. Increased flow was previously shown to result in a linear increase in discharge current. ${ }^{5}$

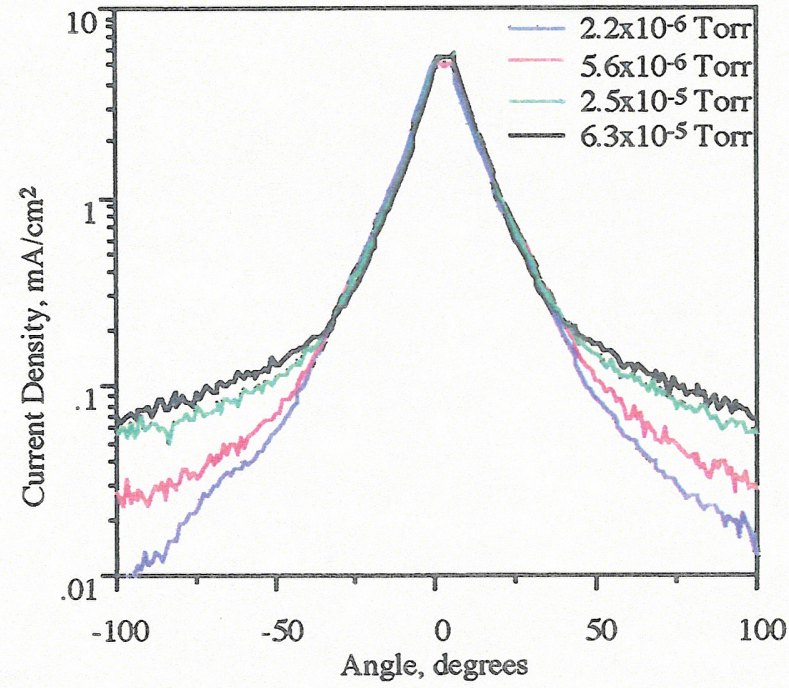

Figure 5: Ion Current Density Distribution $60 \mathrm{~cm}$ from the SPT-100 as a Function of Facility Pressure

Ingestion of only background xenon gas is insufficient to completely account for this change in discharge current. This was based on a kinetic theory calculation of the one way flux of background neutral xenon into the discharge chamber. This calculation assumed $300 \mathrm{~K}$ xenon atoms at the measured facility pressure with a Maxwellian velocity distribution at the geometric thruster exit area. Neutral xenon from the cathode was thought to be the additional source of ingested neutral xenon even though the cathode flow is directed away from the interior of the discharge chamber. The mean free path for elastic scattering was estimated to be on the order of a centimeter. This was sufficiently small as to allow a significant number of neutral xenon atoms from the cathode to be redirected into the $10 \mathrm{~cm}$ diameter discharge chamber. Approximately one fourth of the nominal cathode flow would need to have been ingested to compliment the background xenon ingested to completely account for the $6 \%$ increase in discharge current at $6 \times 10^{-5}$ Torr.

As the facility pressure was increased the ion current density at large angles increased. The ion current density in the central core was unaffected. During this test it was not possible to reach a facility pressure low enough to have no effect on the ion beam density distribution.

The increase in ion current density at large angles with increasing facility pressure was attributed to charge exchange. Charge exchange occurring between fast moving directed ions and slow moving background xenon atoms, moving in arbitrary directions, resulted in șlow ions, moving in arbitrary directions. The probability of charge exchange was greatest within the central core where the ion density was large and at elevated facility pressures where the background atom number density was increased. For non-negligible facility pressures some of the slow moving charge exchange ions created in the core were subsequently measured at large angles. Because charge exchange with a background gas would be minimal on orbit, interpretation of ground test data for assessment of spacecraft integration issues must include consideration of this effect.

The ion current density distribution measured for each of the three $T-100$ stationary plasma thrusters is shown in Figure 6. As mentioned previously, the later versions of this thruster had claimed improvements that were not externally visible aind were thought to be changes in the shape and strength of the applied magnetic field.

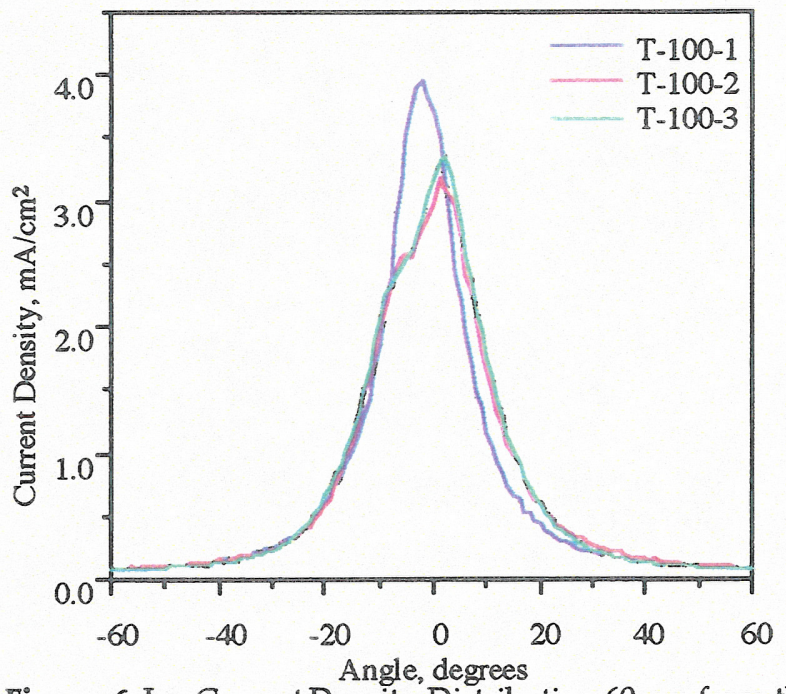

Figure 6: Ion Current Density Distribution $60 \mathrm{~cm}$ from the exit of the T-100-1, T-100-2, and T-100-3.

There were differences in the performance of these thrusters. The first generation thruster, which had the highest peak ion current density, had a measured thrust of $78.5 \mathrm{mN}$. The second and third generation thrusters, which had comparable ion current density distributions, had measured thrusts of 83 and $82.5 \mathrm{mN}$, respectively. These data suggested that the second and third generation 
thrusters have a higher average ion energy than the first generation thruster. Each distribution was asymmetric with respect to the geometric thruster axis. This implied that the thrust vector did not coincide with the geometric thruster axis, giving rise to additional spacecraft integration issues.

\section{Off-Nominal Operating Condition Data:}

As previously indicated, off-nominal performance conditions were tested to determine how the ion current density distribution varied with operating conditions. The effect of discharge voltage, discharge current, cathode flow rate, and magnetic field strength were considered. Measurements were taken with each type of thruster. However, the T-100 data were very similar to SPT-100 data and will not be presented.

Figure 7 shows the variation in ion current

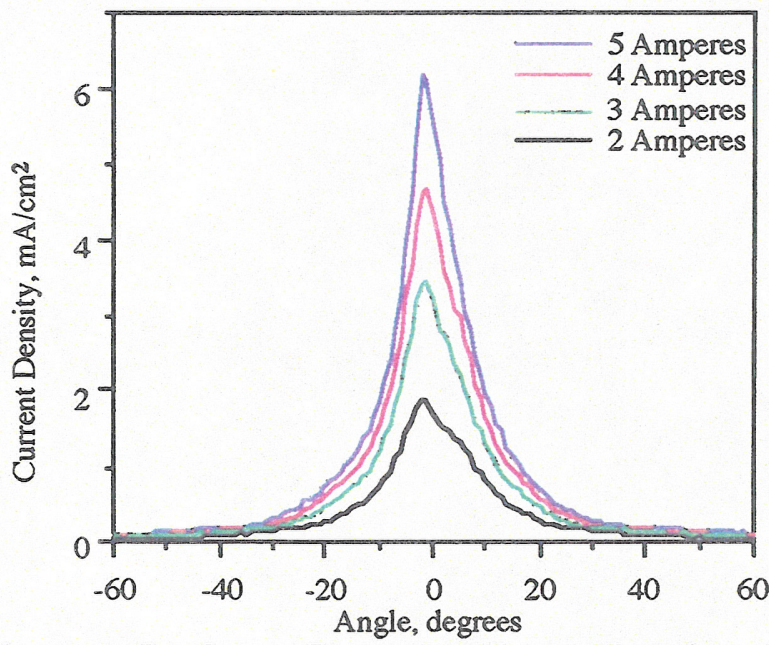

Figure 7a: Ion Current Density Distribution $60 \mathrm{~cm}$ from the SPT-100 Exit Plane as a Function of Discharge Current, 300 Volts Discharge Voltage.

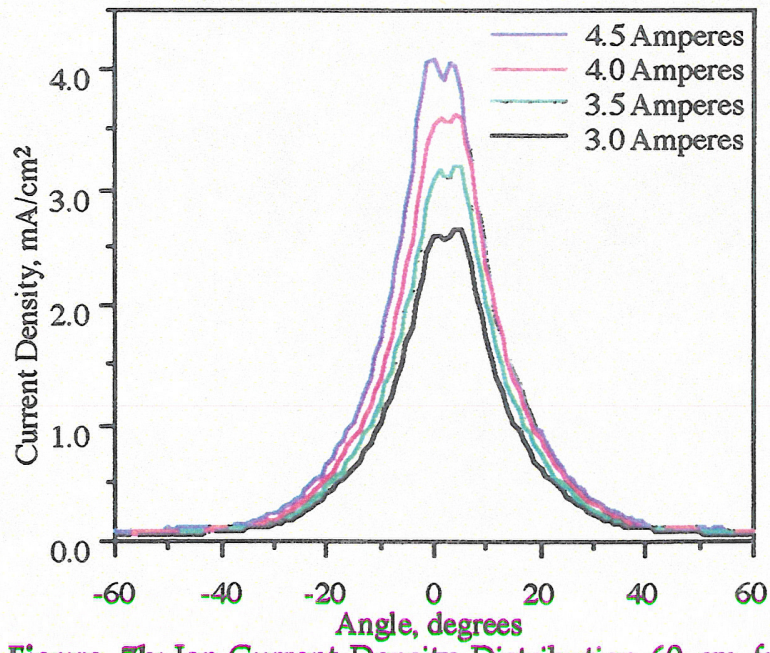

Figure 7b: Ion Current Density Distribution $60 \mathrm{~cm}$ from the Exit Plane of the D-55 as a Function of Discharge Current, 300 Vol ts Discharge Voltage. density distribution as a function of discharge current for both the SPT-100 and the TAL D-55. In each case the ion current density varied linearly with discharge current. Because the discharge current varied linearly with anode flow rate the change in ion current density was attributed to changes in mass flow rate.

The variation of ion-beam current density distribution with discharge voltage, at a constant four Amperes discharge current, is shown for the SPT-100 and TAL D-55 in Figure 8. For the SPT-100 the ion current increased as the discharge voltage was increased until a discharge voltage of 400 Volts was reached. Further increase of discharge voltage above 400 Volts led to a reduction in ion beam current. For the TAL D-55 the ion current increased monotonically with discharge voltage.

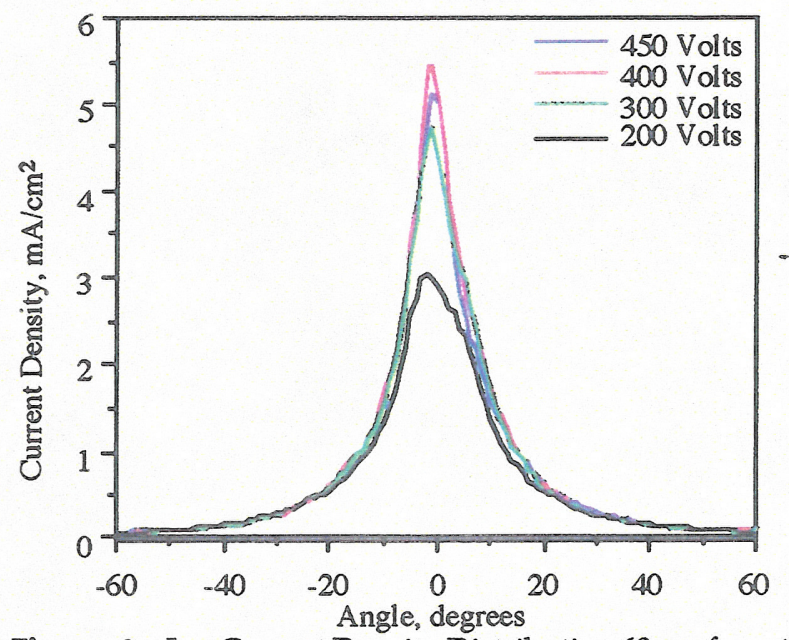

Figure 8a: Ion Current Density Distribution $60 \mathrm{~cm}$ from the SPT-100 Exit Plane as a Function of Discharge Voltage, 4 Amperes Discharge Current.

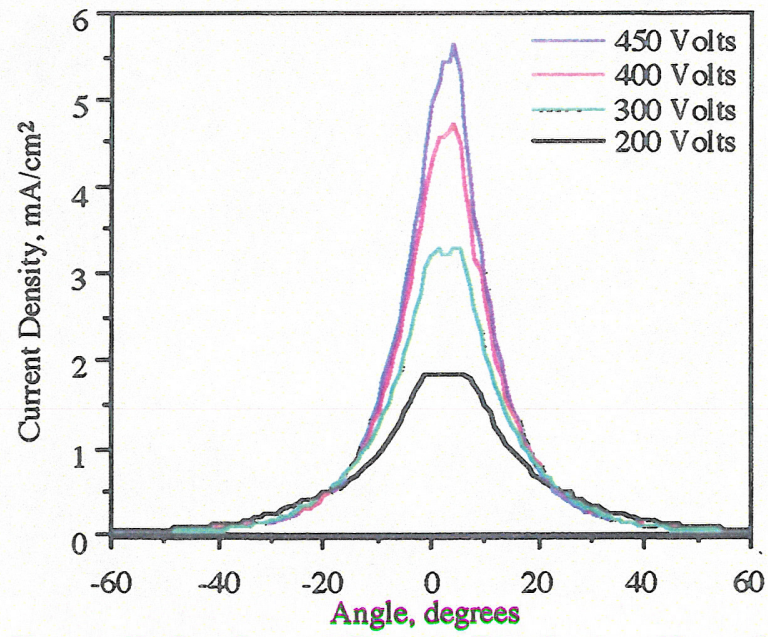

Figure 8b: Ion Current Density Distribution $60 \mathrm{~cm}$ from the D-55 Exit Plane as a Function of Discharge Voltage, 4 Amperes Discharge Current. 
The mass flow rate for all the distributions taken with a particular thruster was essentially constant since the discharge current was constant. Therefore, changes in the total ion beam current, determined by integrating the ion current density distribution, reflected changes in the fraction of the flow which was ionized and the ratio of singly charged ions to multiply charged ions. The data indicated that at higher discharge voltages multiply charged ions dominated. This was also implied by performance measurements. Thrust increased with discharge voltage at a constant discharge current (mass flow rate). At higher discharge voltages, however, the thrust begins to approach an asymptotic limit. The inability to effectively further increase thrust is attributed to the decreased efficiency in producing multiply charged ions. These efficiency losses, however, are insufficient for explaining the reduction in ion beam current as discharge voltage was increased from 400 to 450 Volts in the SPT- 100 . This phenomenon is currently unexplained.

Several other thruster variables were considered. The effect of cathode flow rate on the ion current density distribution was evaluated for the TAL. Increasing cathode flow rate resulted in a slight increase in peak ion current density. It should be noted that the total cathode flow rate was considerably higher for this thruster than for the others, due to the fact that it was a laboratory model thruster. These measurements were not conducted with the SPT since this thruster used an internal flow splitter to divide the flow between the cathode and anode.

The effect of magnetic field strength was also considered. This parameter had a negligible effect on the SPT-100 and T-100 ion current density distribution and a slight effect on the TAL ion current density distribution. Changes in the magnetic field shape by varying the relative strength of the inner and outer electromagnets resulted in a more pronounced, but still small, change in ion current density distribution for the TAL. These measurements were not conducted for the SPT-100 and T-100 because the inner and outer electromagnets were internally connected in series.

\section{Conclusions}

The distribution of ion current density as a function of angle from the thruster axis for each of five different Hall thrusters at both nominal operating conditions and off-nominal operating conditions was measured. These data showed that the ion current density distribution of the SPT-100, the T-100, and the TAL D-55 operating at nominal operating conditions were very similar. These data indicated that multiply charged ions were a significant minority in the exhausting plasma for each of these thrusters operating at nominal operating conditions. There was no statistically significant difference between the ion current density distributions measured using a new SPT-100 and using a SPT-100, that had completed a 6000 hour wear test.

The effect of facility pressure on the ion current density distribution was found to be significant at the pressures tested. Ion beam current was found to vary linearly with discharge current. The effect of discharge voltage on ion current density indicated increased multiply charged ions at higher discharge voltages. The effects of cathode flow rate and magnetic field strength on the ion current density distribution were found to be small. These data will permit spacecraft integrators to extrapolate previous experimental measurements of possible thruster plume/spacecraft interactions to any given spacecraft configuration.

\section{Acknowledgment}

The authors would like to gratefully acknowledge the support of Dr. Leonard Caveny of the Ballistic Missile Defense Organization's Office of Innovative Science and Technology.

\section{References}

1. Brophy, J.R., Barnett, J.W., Sankovic, J.M., and Barnhart, D.A., "Performance of the Stationary Plasma Thruster: SPT-100," AIAA92-3155, July 1992.

2. Randolph, T., Pencil, E., and Manzella, D., "Far-Field Plume Contamination and Sputtering of the Stationary Plasma Thruster," ALAA-94-2855, June 1994.

3. Dickens, J., Mandowski, J., Kristiansen, M., and O'Hair, E., "Impact of Hall Thrusters on Communication System Phase Noise," AIAA95-2929, July 1995.

4. Absalamov, S.K., et. al., "Measurements of the Plasma Parameters in the Stationary Plasma Thruster (SPT-100) Plume and its Effect on Spacecraft Components," AIAA-92-3156, July 1992.

5. Sankovic, J.M., Hamley, J.A., and Haag, T.W., "Performance Evaluation of the Russian SPT100 Thruster at NASA LeRC," IEPC-93-094, Sept. 1993.

6. Garner, C., Polk, J., and Brophy, J., "Cyclic Endurance Test of a SPT-100 Stationary Plasma Thruster," AIAA-94-2856, June 1994. 
7. Sankovic, J.M., Haag, T.W., and Manzella, D.H., "Operating Characteristics of the Russian D-55 Thruster with Anode Layer," AIAA-94-3011, June 1994.

8. Fischer, G., et. al., "Design of a High Efficiency Power Processor for the Russian Stationary Plasma Thruster," AIAA-93-043, June 1993.

9. Manzella, D.H., "Stationary Plasma Thruster Plume Emissions," IEPC-93-097, Sept. 1993.

10. Manzella, D.H., "Stationary Plasma Thruster Ion Velocity Distribution," IEPC-94-3141, Aug. 1994.

11. Bishaev, A.M. and Kim, V., "Local Plasma Properties in a Hall-Current Accelerator with an Extended Acceleration Zone," Sov. Phys. Tech. Phys. Vol 23, No.9, Sept. 1978, 1055-1057.

\section{Appendix}

Because the probe rotates about a point, consider describing a differential surface element in cylindrical coordinates, ie:

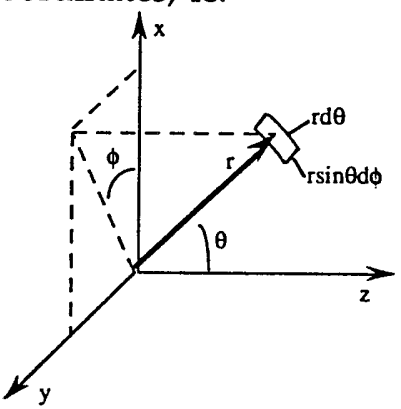

Figure A1: Differential Surface Element

The thruster fires along the $z$-axis with its exit plane in the xy plane. The total ion current passing throught the forward hemisphere can be calculated by integration.

$$
I_{\text {tot }}=\int_{0}^{\pi / 2} \int_{0}^{2 \pi} i(\phi, \theta) r \sin \theta d \phi r d \theta
$$

Assuming the ion beam is axisymmetrix about the zaxis $i(\phi, \theta)=i(\theta)$ and therefore:

$$
\mathrm{I}_{\text {tot }}=\mathrm{r}^{2} \int_{0}^{2 \pi} d \phi \int_{0}^{\pi / 2} \mathrm{i}(\theta) \sin \theta d \theta
$$

Further simplification results in the following expression that can be evaluated directly based on the experiemntal data:

$$
I_{\text {tot }}=2 \pi r^{2} \int_{0}^{\pi / 2} i(\theta) \sin \theta d \theta
$$




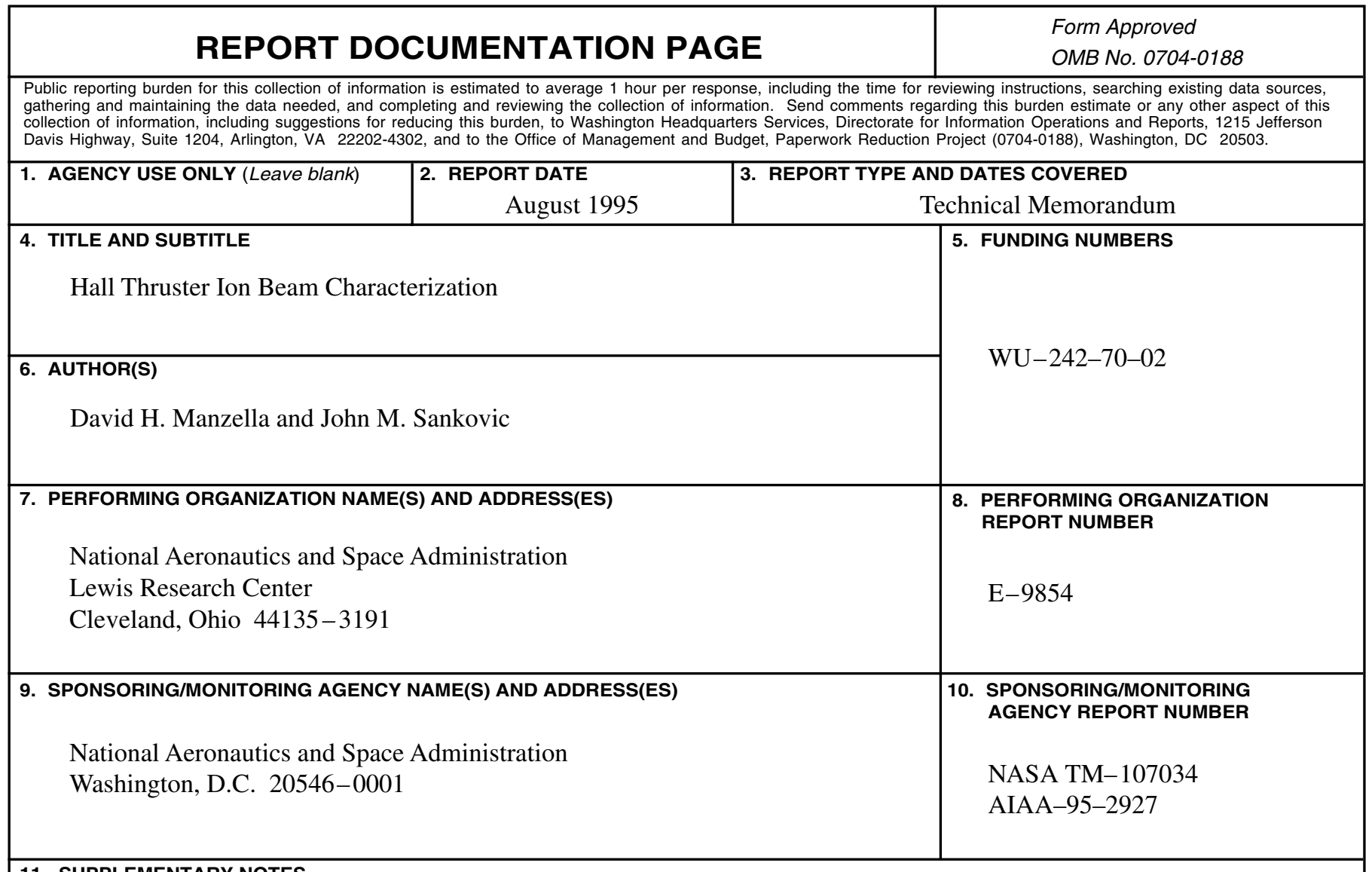

\section{SUPPLEMENTARY NOTES}

Prepared for the 31st Joint Propulsion Conference and Exhibit cosponsored by AIAA, ASME, SAE, and ASEE, San Diego, California, July 10-12, 1995. David H. Manzella, NYMA, Inc., 2001 Aerospace Parkway, Brook Park, Ohio 44142 (work funded by NASA Contract NAS3-27186) and John M. Sankovic, Lewis Research Center. Responsible person, John M. Sankovic, organization code 5330, (216) 433-7429.

\begin{tabular}{|l|l|}
\hline 12a. DISTRIBUTION/AVAILABILITY STATEMENT & 12b. DISTRIBUTION CODE
\end{tabular}

Unclassified - Unlimited

Subject Category 20

This publication is available from the NASA Center for Aerospace Information, (301) 621-0390.

13. ABSTRACT (Maximum 200 words)

The distribution of ion current density as a function of angle from the thruster axis was measured at nominal operating conditions of 300 Volts discharge voltage and 4.5 Amperes discharge current for two SPT-100 stationary plasma thrusters from Fakel Enterprises, a D-55 anode layer thruster from the Central Scientific Research Institute for Machine Building (TsNIIMASH), and a first, second, and third generation T-100 stationary plasma thruster from the Scientific Research Institute of Thermal Process (NIITP). The data showed that the current density distributions of these thrusters were similar. Some differences in peak ion current density were observed. Multiply charged ions were found to be a small fraction of the plasma plume for all of the thrusters. The effect of facility pressure on ion current density distribution was found to be non-negligible at pressures above $2 \times 10^{-6}$ Torr. The ion current density distributions of a new SPT-100 and a 6000 hour wear tested SPT-100 exhibited no discernible difference. Ion current density measurements were also taken at off-nominal thruster operating conditions.

\begin{tabular}{|l|l|l|}
\hline $\begin{array}{l}\text { 14. SUBJECT TERMS } \\
\text { Stationary plasma thruster; Anode layer thruster }\end{array}$ & $\begin{array}{c}\text { 19. SECURITY CLASSIFICATION } \\
\text { OF ABSTRACT } \\
\text { Unclassified }\end{array}$ \\
$\begin{array}{c}\text { 17. SECURITY CLASSIFICATION } \\
\text { OF REPORT } \\
\text { Unclassified }\end{array}$ & $\begin{array}{c}\text { 18. SECURITY CLASSIFICATION } \\
\text { OF THIS PAGE } \\
\text { Unclassified }\end{array}$ & \begin{tabular}{c} 
Undied \\
\hline
\end{tabular}
\end{tabular}

